\title{
Investigation of the Interaction Between Novel Spiro Thiazolo[3,2-a][1,3,5]Triazines and Bovine Serum Albumin by Spectroscopic Methods
}

\author{
Ying Yang $\cdot$ Xianyong Yu $\cdot$ Wenhua Tong $\cdot$ Shiyu Lu \\ Heting Liu $\cdot$ Qin Yao $\cdot$ Hu Zhou
}

Received: 23 February 2012/ Accepted: 11 August 2012/Published online: 19 March 2013

(C) Springer Science+Business Media New York 2013

\begin{abstract}
The interaction between novel spiro thiazolo[3,2-a][1,3,5]triazines (NSTT) and bovine serum albumin (BSA) was investigated using fluorescence and ultraviolet spectroscopy at different temperatures $(302$ and $310 \mathrm{~K})$ under imitated physiological conditions. The experimental results show that the fluorescence quenching mechanism between NSTT and BSA is by a static quenching mechanism. The binding constant $\left(K_{\mathrm{a}}\right)$ and number of binding sites $(n)$ between NSTT and BSA at different temperatures were obtained. Negative values of $\Delta G^{\circ}, \Delta H^{\circ}$, and $\Delta S^{\circ}$ indicate that the interaction between NSTT and BSA is driven by hydrogen bonds and van der Waals forces. Using the Förster nonradiation energy transfer theory, the binding distance between BSA and NSTT was calculated. These results provide valuable information on the interaction between NSTT and BSA as well as the influence of substituent groups on the interaction.
\end{abstract}

Keywords Novel spiro thiazolo[3,2-a][1,3,5]triazines · Bovine serum albumin * Fluorescence spectroscopy $\cdot$ Absorption spectroscopy $\cdot$ Interaction

\section{Introduction}

Derivatives of spiropyrrolidines are found in a variety of biological applications such as antimicrobial, antitumoral, and antibiotic agents, and inhibitors of the human NK-1

Y. Yang $\cdot$ X. Yu $(\bowtie) \cdot$ S. Lu $\cdot$ H. Liu $\cdot$ Q. Yao $\cdot$ H. Zhou $(\bowtie)$

Key Laboratory of Theoretical Chemistry and Molecular Simulation of Ministry of Education, Hunan Province College Key Laboratory of QSAR/QSPR, School of Chemistry and Chemical Engineering, Hunan University of Science and Technology, Xiangtan 411201, China

e-mail: yuxianyong1975@163.com

H. Zhou

e-mail: zhouhu2006@163.com

W. Tong

Department of Chemical Engineering, Qingdao Institute of Bioenergy and Bioprocess Technology, Chinese Academy of Sciences, Qingdao 266101, China 
Scheme 1 Structures of novel spiro thiazolo[3,2-a] $[1,3,5]$ triazines analogues

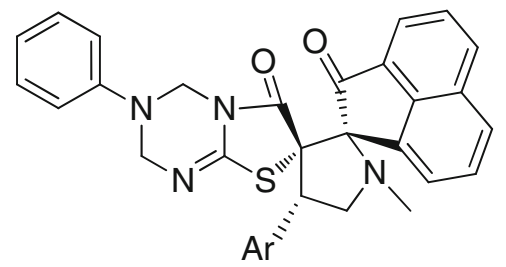

Ar: $1 \mathrm{C}_{6} \mathrm{H}_{5}$;

$24-\mathrm{CH}_{3} \mathrm{C}_{6} \mathrm{H}_{4}$;

3 4- $\mathrm{ClC}_{6} \mathrm{H}_{4}$;

4 4- $\mathrm{FC}_{6} \mathrm{H}_{4}$;

$52,4-\mathrm{Cl}_{2} \mathrm{C}_{6} \mathrm{H}_{3}$

receptor. Spiropyrrolidines are also motifs in some pharmacologically active alkaloids, such as rhynchophylline, corynoxeine, horsifiline, mitraphylline and spirotryprostatins [1-4].

As is well known, serum albumins (SA) contribute to colloid osmotic blood pressure and the maintenance of blood $\mathrm{pH}$. They also play a dominant role in drug disposition and efficacy. Most drugs are transported as a complex with SA, which makes SA an important part of drug metabolism [5-7]. Bovine serum albumin (BSA) is a natural globular protein with a relatively high molecular mass $(66.3 \mathrm{kDa})$ and a single polypeptide chain containing 585 amino acid residues. It consists of three linearly arranged and structurally distinct homologous domains (I-III), which are divided into nine loops (L1-L9). Each domain contains two sub-domains (A and B). Similar to human serum albumin (HSA), BSA contains 17 disulfide bridges and a free thiol group (Cys 34). So, BSA is an appropriate protein model to study the interaction between serum albumins and drugs [8,9].

Studies on the interaction between plasma proteins and drugs have been an important research field in life sciences, chemistry and clinical medicine [10]. In this paper, the binding between novel spiro thiazolo[3,2-a][1,3,5]triazines (NSTT, Scheme 1) and BSA was studied with fluorescence and absorption spectrum under imitated physiological conditions. The binding affinity was strongest for compound $\mathbf{5}$ and ranked in the order $\mathbf{1}<\mathbf{2}<\mathbf{3}<\mathbf{4}<\mathbf{5}$.

\section{Experimental}

\subsection{Reagents}

BSA ( $\geq 99 \%$ ) was obtained from Jitian Bioengineering Co. (Shanghai, China) and was dissolved in a Tris- $\mathrm{HCl}\left(0.10 \mathrm{~mol} \cdot \mathrm{L}^{-1}, \mathrm{pH}=7.4\right)$ buffer to form the BSA solution with a concentration of $1.00 \times 10^{-5} \mathrm{~mol} \cdot \mathrm{L}^{-1}$. A Tris- $\mathrm{HCl}$ buffer $\left(0.10 \mathrm{~mol} \cdot \mathrm{L}^{-1}, \mathrm{pH}=7.4\right)$ containing $0.10 \mathrm{~mol} \cdot \mathrm{L}^{-1} \mathrm{NaCl}$ was selected to keep the $\mathrm{pH}$ constant and to maintain the ionic strength of the solution. Novel spiro thiazolo[3,2-a][1,3,5]triazines were obtained according to the reported procedure [4] and the NSTT $\left(1.00 \times 10^{-3} \mathrm{~mol} \cdot \mathrm{L}^{-1}\right)$ solution was prepared in DMF. All other reagents were of analytical grade and double-distilled water was used in the experiments.

\subsection{Apparatus}

Fluorescence spectra were recorded on a Shimadzu RF-5301 fluorescence spectrophotometer (Tokyo, Japan) with a SB-11 water bath (Eyela) and $1.0 \mathrm{~cm}$ quartz cells. The excitation and emission slits were 5 and $10 \mathrm{~nm}$, respectively. The absorption spectra were obtained from a Shimadzu UV-2501 spectrophotometer (Tokyo, Japan). The pH 
measurements were made with a Leici pHS-2 digital pH-meter (Shanghai, China) with a combined glass-calomel electrode.

\subsection{Procedures}

A $2.5 \mathrm{~mL}$ solution containing $1.00 \times 10^{-5} \mathrm{~mol} \cdot \mathrm{L}^{-1} \mathrm{BSA}$ was titrated by successive additions of $1.00 \times 10^{-3} \mathrm{~mol} \cdot \mathrm{L}^{-1}$ NSTT solution with final concentrations of NSTT ranging from 0 to $3.60 \times 10^{-5} \mathrm{~mol} \cdot \mathrm{L}^{-1}$. Titrations were done manually by using a microinjector. Fluorescence spectra were measured in the range of $280-500 \mathrm{~nm}$ at the excitation wavelength of $280 \mathrm{~nm}$. The fluorescence spectra were measured at 302 and $310 \mathrm{~K}$. The range of synchronous scanning were $\lambda_{\mathrm{ex}}=240, \lambda_{\mathrm{em}(\mathrm{a})}=255, \lambda_{\mathrm{em}(\mathrm{b})}=300 \mathrm{~nm}$, where the differences in the wavelengths $(\Delta \lambda)$ were 15 and $60 \mathrm{~nm}$.

The absorption spectra of BSA in the presence of different concentrations of NSTT were recorded in the range of $200-450 \mathrm{~nm}$ at room temperature. The concentration of BSA was kept at $1.00 \times 10^{-5} \mathrm{~mol} \cdot \mathrm{L}^{-1}$, while that of NSTT was varied from 0 to $2.00 \times 10^{-5} \mathrm{~mol} \cdot \mathrm{L}^{-1}$. The UV-Vis absorption spectra of NSTT solutions at $1.00 \times 10^{-5} \mathrm{~mol} \cdot \mathrm{L}^{-1}$ were measured in the range of $200-500 \mathrm{~nm}$ at $310 \mathrm{~K}$.

\section{Results and Discussion}

\subsection{Fluorescence Quenching of BSA by NSTT}

The fluorescence quenching of NSTT with BSA is shown in Fig. 1. It is apparent from Fig. 1 that BSA has a strong fluorescence emission peak at $340 \mathrm{~nm}$ after being excited and the fluorescence intensity of BSA decreases with increasing concentration of NSTT, which shows that NSTT interacts with BSA and quenches the intrinsic fluorescence of BSA. Furthermore, these phenomena indicate that the high affinity-binding site of BSA is close

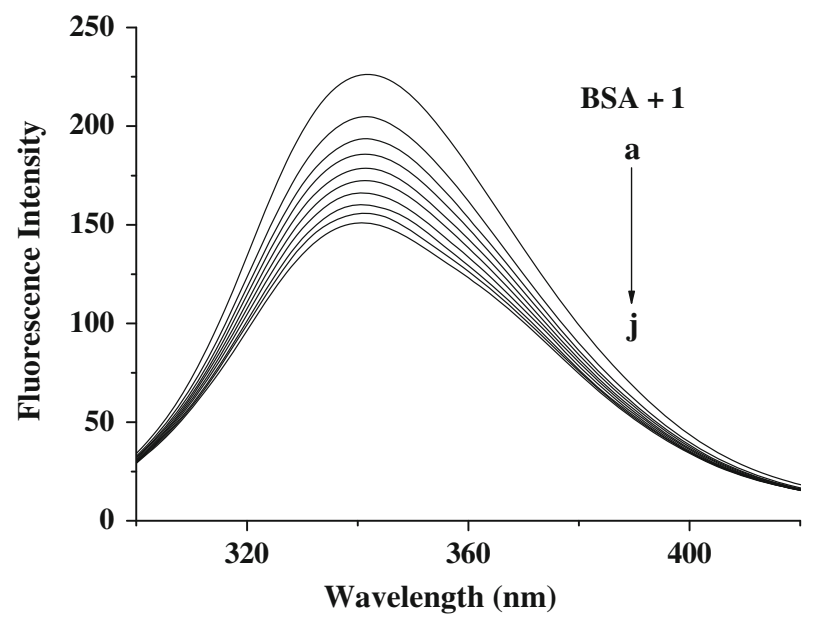

Fig. 1 The fluorescence quenching spectra of BSA by NSTT at $310 \mathrm{~K}$. $\lambda_{\text {ex }}=280 \mathrm{~nm} ; c(\mathrm{BSA})=1.00 \times$ $10^{-5} \mathrm{~mol} \cdot \mathrm{L}^{-1} ; c(\mathrm{NSTT}) /\left(\times 10^{-6} \mathrm{~mol} \cdot \mathrm{L}^{-1}\right)(a-j): 0,4.0,8.0,12.0,16.0,20.0,24.0,28.0,32.0$, and 36.0 
to the Trp and (or) tyrosine of BSA, which suggests that interaction has occurred and energy has been transferred $[11,12]$.

Fluorescent quenching can occur by two different mechanisms, static quenching and dynamic quenching. Dynamic quenching, also known as collisional quenching, results from collisions between fluorophores and a quencher; static quenching results from ground state complex formation between fluorophores and a quencher [13]. The fluorescence quenching data are usually analyzed by the Stern-Volmer equation [14-16]:

$$
\frac{F_{0}}{F}=1+K_{\mathrm{SV}}[\mathrm{Q}]=1+k_{\mathrm{q}} \tau_{0}[\mathrm{Q}]
$$

$F_{0}$ and $F$ are the steady-state fluorescence intensities in the absence and the presence of the quencher (NSTT), respectively. $K_{\mathrm{SV}}$ is the Stern-Volmer quenching constant, and [Q] is the concentration of quencher. $k_{\mathrm{q}}$ is the bimolecular quenching rate constant; $\tau_{0}$ is the average lifetime of the molecule without the quencher and its value is $10^{-8} \mathrm{~s}$ [17]. The Stern-Volmer plots of the quenching of BSA fluorescence by NSTT at $302 \mathrm{~K}$ are shown in Fig. 2. The corresponding correlation coefficients, at two different temperatures, were calculated and are shown in Table 1. As we can see, compound $\mathbf{5}$ has the highest quenching constant and compound $\mathbf{1}$ has the lowest. For dynamic quenching, the maximum scatter collision quenching constant of various quenchers with the biopolymers is $2.0 \times 10^{10} \mathrm{~L} \cdot \mathrm{mol}^{-1} \cdot \mathrm{s}^{-1}$. The results show that $k_{\mathrm{q}}$ is much larger than $2.0 \times 10^{10} \mathrm{~L} \cdot \mathrm{mol}^{-1} \cdot \mathrm{S}^{-1}$, which indicates that the quenching mechanism of BSA-NSTT interactions is initiated by complex formation rather than by dynamic collisions $[18,19]$.

\subsection{UV-Vis Absorption Spectra}

The absorption spectra of BSA in the presence and absence of NSTT (compound 1) were recorded and are shown in Fig. 3. With the addition of NSTT, the absorbance intensity

Fig. 2 Stern-Volmer plots of BSA $\left(1.00 \times 10^{-5} \mathrm{~mol} \cdot \mathrm{L}^{-1}\right)$ quenched by NSTT at $302 \mathrm{~K}$

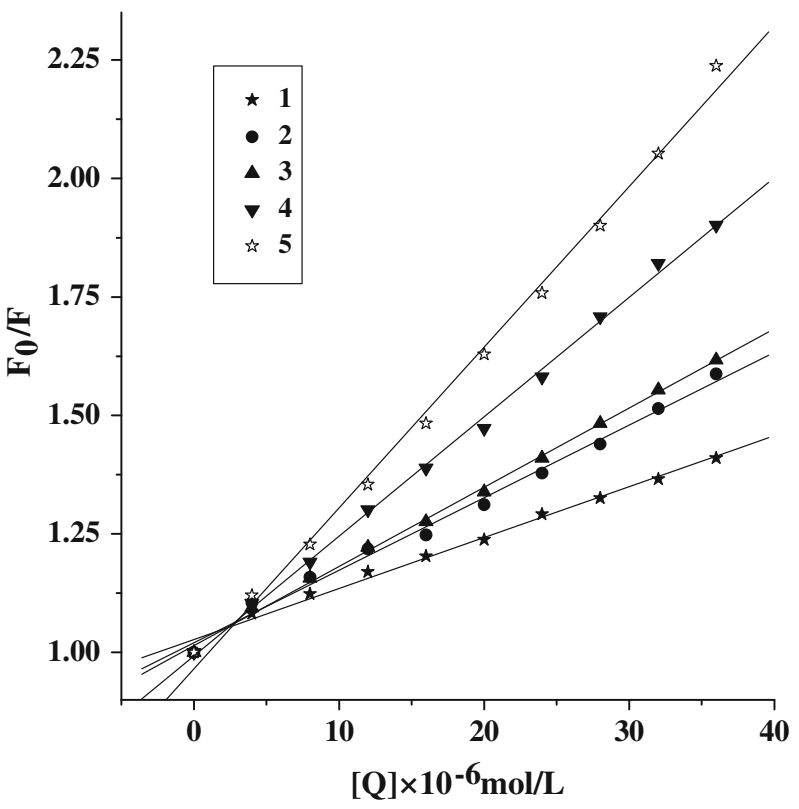


Table 1 The quenching constants of BSA by NSTT at two different temperatures

\begin{tabular}{lllll}
\hline Compound & $T(\mathrm{~K})$ & $K_{\mathrm{SV}}\left(\mathrm{L} \cdot \mathrm{mol}^{-1}\right)$ & $k_{\mathrm{q}}\left(\mathrm{L} \cdot \mathrm{mol}^{-1} \cdot \mathrm{s}^{-1}\right)$ & $R$ \\
\hline $\mathbf{1}$ & 302 & $1.07 \times 10^{4}$ & $1.07 \times 10^{12}$ & 0.9957 \\
$\mathbf{2}$ & 310 & $1.30 \times 10^{4}$ & $1.30 \times 10^{12}$ & 0.9924 \\
& 302 & $1.53 \times 10^{4}$ & $1.53 \times 10^{12}$ & 0.9962 \\
$\mathbf{3}$ & 310 & $1.86 \times 10^{4}$ & $1.86 \times 10^{12}$ & 0.9956 \\
& 302 & $1.67 \times 10^{4}$ & $1.67 \times 10^{12}$ & 0.9990 \\
$\mathbf{4}$ & 310 & $1.43 \times 10^{4}$ & $1.43 \times 10^{12}$ & 0.9969 \\
& 302 & $2.52 \times 10^{4}$ & $2.52 \times 10^{12}$ & 0.9990 \\
$\mathbf{5}$ & 310 & $2.38 \times 10^{4}$ & $2.38 \times 10^{12}$ & 0.9984 \\
& 302 & $3.39 \times 10^{4}$ & $3.39 \times 10^{12}$ & 0.9980 \\
& 310 & $3.23 \times 10^{4}$ & $3.23 \times 10^{12}$ & 0.9977 \\
\hline
\end{tabular}

$R$ is the linear correlation quotient

(A) increases. Furthermore, the absorption maximum shifts. These observations reconfirm that BSA can bind to NSTT and form a BSA-NSTT complex [20, 21].

\subsection{Binding Constant and Binding Sites}

Static quenching is caused by the formation of a non-fluorescent ground state fluorophorequencher complex. If there are $n$ substantive binding sites on the surface of a protein to accommodate the ligand molecules, the equilibrium between free and bound molecule is given as follows $[22,23]$ :

$$
\log _{10} \frac{\left(F_{0}-F\right)}{F}=\log _{10} K_{\mathrm{a}}+n \log _{10}[\mathrm{Q}]
$$

where $K_{\mathrm{a}}$ is the binding constant or the apparent association constant for drug-protein interaction and $n$ is the number of binding sites. The double-logarithm curve $\left(\log _{10}\left[\left(F_{0-} F\right) / F\right]\right.$ versus $\log _{10}$ [NSTT]) is shown in Fig. 4 and Table 2 gives the corresponding calculated results. The results suggest that a strong binding site exists between NSTT and BSA. Moreover, we can conclude that all of the substitutions on the benzene ring enhance the binding affinity of BSA and NSTT. According to Table 2, the binding constants of the interaction between them increase in the following order at both temperatures: $\mathbf{1}<\mathbf{2}<\mathbf{3}<\mathbf{4}<\mathbf{5}$, which means that 5 has the strongest ability to bind with BSA. We suggest that the factors resulting in the binding trends may be: increasing the polarity of substituent group $\left(4-\mathrm{CH}_{3}<4-\mathrm{Cl}<4-\mathrm{F}<2,4-\mathrm{Cl}_{2}\right.$ ) strengthens its binding affinity. For $2,4-\mathrm{Cl}_{2}$, the large-size drug molecule has a larger hydrophobic area that can interact with hydrophobic surface on the protein molecule [24].

\subsection{Thermodynamic Parameters and the Nature of the Binding Forces}

The interaction forces between a small molecule and a macromolecule mainly include hydrogen bonds, van der Waals forces, electrostatic forces and hydrophobic interaction forces. The thermodynamic parameters were evaluated using Eqs. 3, 4 and 5 [25]:

$$
\ln \frac{K_{\mathrm{a} 2}}{K_{\mathrm{a} 1}}=\frac{\Delta H^{\mathrm{o}}}{R}\left(\frac{1}{T_{1}}-\frac{1}{T_{2}}\right)
$$




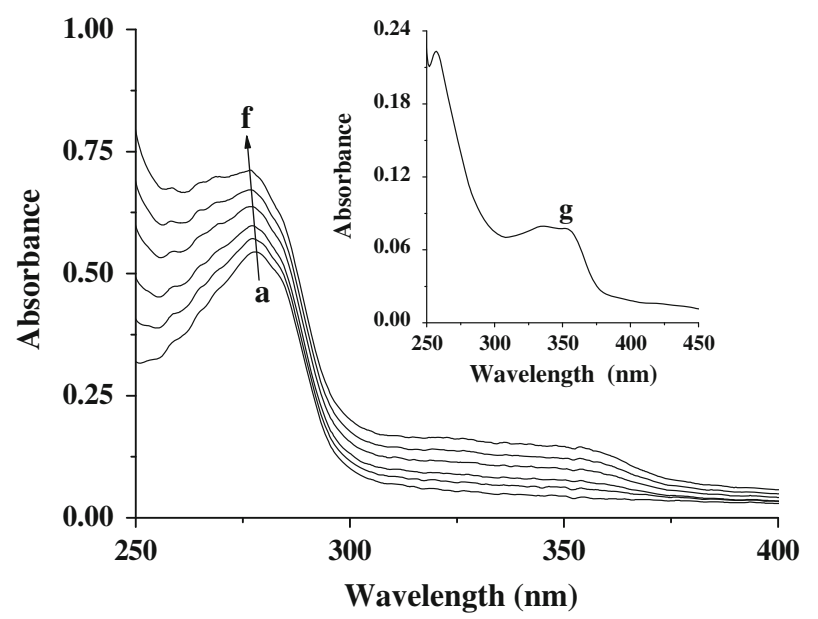

Fig. 3 UV-Vis absorption spectra of BSA in the presence of different concentrations of NSTT (compound 1). $(a-f): c(\mathrm{BSA})=1.00 \times 10^{-5} \mathrm{~mol} \cdot \mathrm{L}^{-1} ; c(\mathrm{NSTT}) /\left(\times 10^{-6} \mathrm{~mol} \cdot \mathrm{L}^{-1}\right)=0.0,4.0,8.0,12.0,16.0$, and 20.0 , respectively; $(g): c(\mathrm{BSA})=0, c(\mathrm{NSTT})=1.00 \times 10^{-5} \mathrm{~mol} \cdot \mathrm{L}^{-1}$

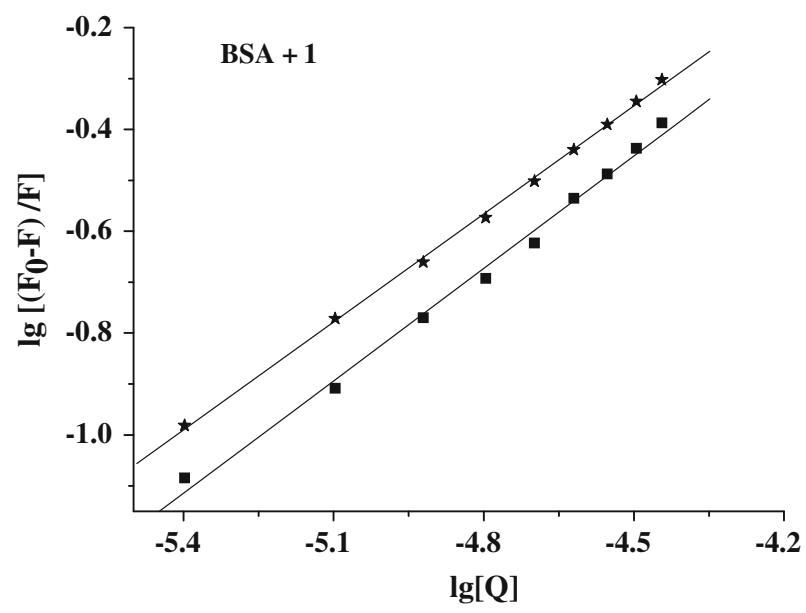

Fig. 4 Plot of $\log _{10}\left[\left(\mathrm{~F}_{0}-\mathrm{F}\right) / \mathrm{F}\right]$ versus $\log _{10}[\mathrm{NSTT}]$ at different temperatures (filed star, $302 \mathrm{~K}$; filled square, $310 \mathrm{~K}$ )

$$
\begin{gathered}
\Delta G^{\circ}=-R T \ln K_{\mathrm{a}} \\
\Delta S^{\circ}=\frac{\Delta H^{\circ}-\Delta G^{\circ}}{T}
\end{gathered}
$$

where $K_{\mathrm{a}}$ is the binding constant at the corresponding temperature $(302,310 \mathrm{~K}), R$ is the gas constant, and $T$ is the experimental temperature. $\Delta H^{\circ}, \Delta G^{\circ}$ and $\Delta S^{\circ}$ are the standard state enthalpy, Gibbs energy and entropy change, respectively. Using the $K_{\mathrm{a}}$ values at two different temperatures, we can calculate the enthalpy change $\left(\Delta H^{\circ}\right)$ and Gibbs energy change $\left(\Delta G^{\circ}\right)$ from Eqs. 3 and 4 and then calculate $\Delta S^{\circ}$ from Eq 5. Their values are listed in Table 3. If $\Delta H^{\circ} \approx 0$ and $\Delta S^{\circ}>0$, the main force is hydrophobic interaction; if $\Delta H^{\circ}<0$ 
Table 2 The binding constants and the number of binding sites for NSTT with BSA at two different temperatures

\begin{tabular}{lllll}
\hline Compound & $T(\mathrm{~K})$ & $K_{\mathrm{a}}\left(\mathrm{L} \cdot \mathrm{mol}^{-1}\right)$ & $n$ & $R$ \\
\hline $\mathbf{1}$ & 302 & $7.25 \times 10^{2}$ & 0.74 & 0.9964 \\
& 310 & $5.76 \times 10^{2}$ & 0.71 & 0.9994 \\
$\mathbf{2}$ & 302 & $1.61 \times 10^{3}$ & 0.78 & 0.9910 \\
$\mathbf{3}$ & 310 & $1.01 \times 10^{3}$ & 0.72 & 0.9920 \\
& 302 & $3.98 \times 10^{3}$ & 0.86 & 0.9975 \\
$\mathbf{4}$ & 310 & $1.35 \times 10^{3}$ & 0.77 & 0.9898 \\
& 302 & $2.36 \times 10^{4}$ & 0.99 & 0.9989 \\
$\mathbf{5}$ & 310 & $1.46 \times 10^{4}$ & 0.95 & 0.9979 \\
& 302 & $6.34 \times 10^{4}$ & 1.06 & 0.9989 \\
& 310 & $2.16 \times 10^{4}$ & 0.97 & 0.9971 \\
\hline
\end{tabular}

$R$ is the linear quotient

Table 3 The thermodynamic parameters of the interaction between BSA and NSTT

\begin{tabular}{lcccc}
\hline Compound & $T(\mathrm{~K})$ & $\begin{array}{l}\Delta H^{\circ} \\
\left(\mathrm{kJ} \cdot \mathrm{mol}^{-1}\right)\end{array}$ & $\begin{array}{l}\Delta G^{\circ} \\
\left(\mathrm{kJ} \cdot \mathrm{mol}^{-1}\right)\end{array}$ & $\begin{array}{l}\Delta S^{\circ} \\
\left(\mathrm{J} \cdot \mathrm{mol}^{-1} \cdot \mathrm{K}^{-1}\right)\end{array}$ \\
\hline $\mathbf{1}$ & 302 & -22.39 & -16.54 & -19.36 \\
$\mathbf{2}$ & 310 & -44.35 & -16.38 & -88.77 \\
$\mathbf{3}$ & 302 & & -18.54 & -278.1 \\
& 310 & -104.82 & -17.83 & -71.71 \\
$\mathbf{4}$ & 302 & -46.94 & -18.59 & \\
& 310 & & -25.28 & -255.5 \\
$\mathbf{5}$ & 302 & -104.93 & -24.71 & \\
\hline
\end{tabular}

and $\Delta S^{\circ}>0$, the main force is the electrostatic effect; if $\Delta H^{\circ}<0$ and $\Delta H^{\circ}<0$, Van der Waals and hydrogen bond interactions play a major role in the reaction. The negative value of $\Delta G^{\circ}$ reveals that the binding process is spontaneous; the negative values of $\Delta H^{\circ}$ and $\Delta S^{\circ}$ indicate that the hydrogen bonds and van der Waals forces are the main forces between the NSTT and BSA [26, 27].

\subsection{Energy Transfer Between BSA and NSTT}

The importance of the energy transfer in biochemistry is that the efficiency of transfer can be used to evaluate the distance between the ligand and the tryptophan residues in the protein [28]. The overlap of the UV absorption spectra of NSTT with the fluorescence emission spectra of BSA is shown in Fig. 5. According to Förster's theory of molecular resonance energy transfer, the distance, $r$, between NSTT and BSA, and the efficiency, $E$, of energy transfer between the donor and acceptor, can be calculated as follows [29-31]:

$$
E=1-\left(\frac{F}{F_{0}}\right)=\frac{R_{0}{ }^{6}}{R_{0}{ }^{6}+r^{6}}
$$


Fig. 5 The overlap of fluorescence emission spectrum of BSA $(a)$ and absorption spectrum of NSTT, $(b) c$ (BSA) $=$ $c(\mathrm{NSTT})=1.00 \times 10^{-5} \mathrm{~mol} \cdot \mathrm{L}^{-1}$; $T=310 \mathrm{~K}$

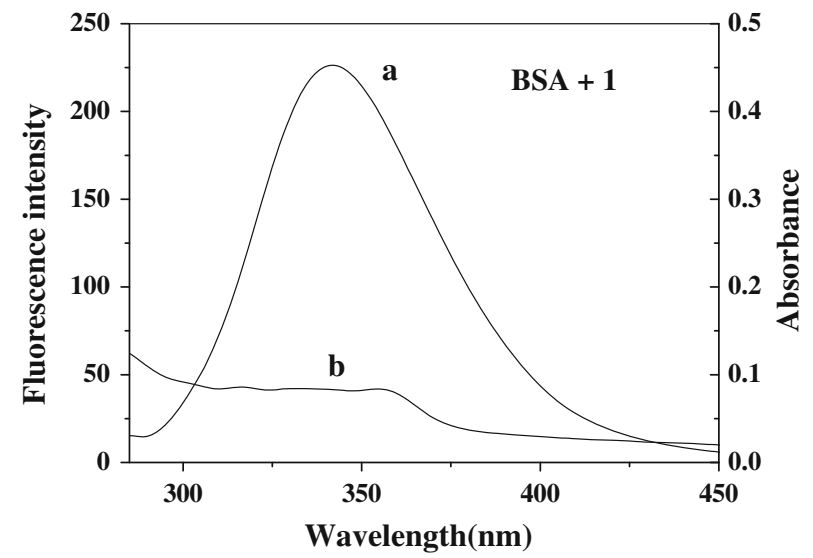

Table 4 The distance parameters between NSTT and BSA at $310 \mathrm{~K}$

\begin{tabular}{lllll}
\hline System & $J\left(\mathrm{~cm}^{3} \cdot \mathrm{L} \cdot \mathrm{mol}^{-1}\right)$ & $R_{0}(\mathrm{~nm})$ & $E$ & $r(\mathrm{~nm})$ \\
\hline BSA+ 1 & $9.77 \times 10^{-15}$ & 2.44 & 0.13 & 3.37 \\
BSA+ 2 & $1.37 \times 10^{-14}$ & 2.58 & 0.15 & 3.44 \\
BSA+ 3 & $8.93 \times 10^{-15}$ & 2.41 & 0.16 & 3.18 \\
BSA+ 4 & $1.09 \times 10^{-14}$ & 2.49 & 0.20 & 3.15 \\
BSA+ 5 & $1.07 \times 10^{-14}$ & 2.48 & 0.23 & 3.04 \\
\hline
\end{tabular}

where $E$ is the efficiency of transfer between the donor and the acceptor and $R_{0}$ is the critical distance where the efficiency of transfer is $50 \% . F_{0}$ and $F$ are the fluorescence intensities of BSA in the absence of and in the presence of the quencher (NSTT), respectively.

$$
J=\frac{F(\lambda) \varepsilon(\lambda) \lambda^{4} \Delta \lambda}{\sum F(\lambda) \Delta \lambda}
$$

$F(\lambda)$ is the fluorescence intensity of the fluorescence donor at wavelength $\lambda$ and $\varepsilon(\lambda)$ is the molar absorption coefficient of the acceptor at wavelength $\lambda$.

$$
R_{0}{ }^{6}=8.8 \times 10^{-25} K^{2} N^{-4} \phi J
$$

In Eq. $8, K^{2}$ is the spatial orientation factor of the dipole, $N$ is the refractive index of the medium, and $\phi$ is the fluorescence quantum yield of the donor. In this case, $K^{2}=2 / 3$, $N=1.336$ and $\phi=0.118$ [32]. The $J, R_{0}, E$ and $r$ were calculated and are presented in Table 4 . These values suggest that the energy transfer from BSA to NSTT occurs with high probability, because $r$ is much $<7 \mathrm{~nm}$. So, the static quenching mechanism caused by binding of NSTT with BSA is responsible for fluorescence quenching [33, 34].

\section{Conclusions}

The interaction between five compounds of NSTT and BSA has been investigated by fluorescence and UV-Vis spectrometry. We conclude that NSTT quenches the fluorescence of BSA by the static quenching procedure. The effects of NSTT with five different 
substituting groups on the binding between the benzene ring and BSA were discussed and the results suggest that all of the substituents can enhance the binding affinity. Because of the differences in the polarity and the volume of different substituting groups, the effect of 2,4- $\mathrm{Cl}_{2}$ is the strongest among them and $4-\mathrm{CH}_{3}$ is the weakest. The experiment results are useful in studying the pharmacological response of drugs and design of dosage forms.

Acknowledgments This work was supported by National Natural Science Foundation of China (20803020, 21006022), Scientific Research Fund of Hunan Provincial Education Department (12K101), The Opening Project of State Key Laboratory of Physical Chemistry of Solid Surfaces (Xiamen University, No.201309), Open Project Program of Key Laboratory of Theoretical Chemistry and Molecular Simulation of Ministry of Education (Hunan University of Science and Technology), and Innovation fund for graduate students of Hunan University of Science and Technology (S110122, S110120).

\section{References}

1. Cui, H., Kakeya, C.-B., Okada, G., Onose, R., Osada, H.: Novel mammalian cell cycle inhibitors, tryprostatins A, B and other diketopiperazines produced by Aspergillus fumigatus. I. Taxonomy, fermentation, isolation and biological properties. J. Antibiot. 49, 527-533 (1996)

2. Cui, H., Kakeya, C.-B., Osada, H.: Novel mammalian cell cycle inhibitors, cyclotryprostatins A-D, produced by Aspergillus fumigatus, which inhibit mammalian cell cycle at G2/M phase. Tetrahedron 53, 59-72 (1997)

3. Edmondson, S., Danishefsky, S.J., Sepp-Lorenzino, L., Rosen, N.: Total synthesis of spirotryprostatin A, leading to the discovery of some biologically promising analogues. J. Am. Chem. Soc. 121, 2147-2155 (1999)

4. Li, X.F., Liu, H.C., Zheng, A.T., Li, Z.K., Li, G.B., Yu, X.Y., Yi, P.G.: Synthesis of novel spiro thiazolo[3,2-a][1,3,5]triazines via 1,3-dipolar cycloaddition of azomethine ylide. Chin. J. Chem. 29, 97-101 (2011)

5. Liu, B., Guo, Y., Wang, J., Xu, R., Wang, X., Wang, D., Zhang, L.Q., Xu, Y.N.: Spectroscopic studies on the interaction and sonodynamic damage of neutral red (NR) to bovine serum albumin (BSA). J. Lumin. 130, 1036-1043 (2010)

6. Hu, Y.J., Liu, Y., Zhang, L.-X., Zhao, R.M., Qu, S.S.: Studies of interaction between colchicine and bovine serum albumin by fluorescence quenching method. J. Mol. Struct. 750, 174-178 (2005)

7. Sułkowska, A., Rownicka, J., Bojko, B., Pozycka, J., Zubik-Skupien, I., Sułkowski, W.: Effect of guanidine hydrochloride on bovine serum albumin complex with antithyroid drugs: fluorescence study. J. Mol. Struct. 704, 291-295 (2004)

8. Tian, J.N., Liu, J.Q., Tian, X., Hu, Z.D., Chen, X.G.: Study of the interaction of kaempferol with bovine serum albumin. J. Mol. Struct. 691, 197-202 (2004)

9. Kun, R., Kis, L., Dékány, I.: Hydrophobization of bovine serum albumin with cationic surfactants with different hydrophobic chain length. Colloids Surf. B 79, 61-68 (2010)

10. Tian, M.Y., Zhang, X.F., Xie, L., Xiang, J.F., Tang, Y.L., Zhao, C.Q.: The effect of $\mathrm{Cu}^{2+}$ on the interaction between an antitumor drug-mitoxantrone and human serum albumin. J. Mol. Struct. 892, 20-24 (2008)

11. Lu, X.L., Fan, J.J., Liu, Y., Hou, A.X.: Characterization of the interaction between cationic erbium(III)porphyrin complex with bovine serum albumin. J. Mol. Struct. 934, 1-8 (2009)

12. Kathiravan, A., Chandramohan, M., Renganathan, R., Sekar, S.: Spectroscopic studies on the interaction between phycocyanin and bovine serum albumin. J. Mol. Struct. 919, 210-214 (2009)

13. Wang, F., Dai, Z.X.: Spectroscopic investigation of the interaction between riboflavin and bovine serum albumin. J. Mol. Struct. 875, 509-514 (2008)

14. Li, J.C., Li, N., Wu, Q.H., Wang, Z., Ma, J.J., Wang, C., Zhang, L.J.: Study on the interaction between clozapine and bovine serum albumin. J. Mol. Struct. 833, 184-188 (2007)

15. Hu, Y.J., Liu, Y., Wang, J.B., Xiao, X.H., Qu, S.S.: Study of the interaction between monoammonium glycyrrhizinate and bovine serum albumin. J. Pharm. Biomed. Anal. 36, 915-919 (2004)

16. Qu, P., Lu, H., Ding, X.Y., Tao, Y., Lu, Z.H.: Study on the interaction of 6-thioguanine with bovine serum albumin by spectroscopic techniques. J. Mol. Struct. 920, 172-177 (2009)

17. Hu, Y.J., Liu, Y., Jiang, W., Zhao, R.M., Qu, S.S.: Fluorometric investigation of the interaction of bovine serum albumin with surfactants and 6-mercaptopurine. J. Photochem. Photobiol. B 80, 235-242 (2005) 
18. Wang, T.H., Zhao, Z.M., Wei, B.Z., Zhang, L., Ji, L.: Spectroscopic investigations on the binding of dibazol to bovine serum albumin. J. Mol. Struct. 970, 128-133 (2010)

19. He, L.L., Wang, X., Liu, B., Wang, J., Sun, Y.G.: Interaction between ranitidine hydrochloride and bovine serum albumin in aqueous solution. J. Solution Chem. 39, 654-664 (2010)

20. Ding, F., Huang, J.L., Lin, J., Li, Z.Y., Liu, F., Jiang, Z.Q., Sun, Y.: A study of the binding of C.I. Mordant Red 3 with bovine serum albumin using fluorescence spectroscopy. Dyes Pigment 82, 65-70 (2009)

21. Wang, T.H., Zhao, Z.M., Zhang, L., Ji, L.: Spectroscopic studies on the interaction between troxerutin and bovine serum albumin. J. Mol. Struct. 937, 65-69 (2009)

22. Naik, P.N., Chimatadar, S.A., Nandibewoor, S.T.: Study on the interaction between antibacterial drug and bovine serum albumin: A spectroscopic approach. Spectrochim. Acta A 73, 841-845 (2009)

23. Wang, C.X., Yan, F.F., Zhang, Y.X., Ye, L.: Spectroscopic investigation of the interaction between rifabutin and bovine serum albumin. J. Photochem. Photobiol. A 192, 23-28 (2007)

24. Kamat, B.P., Seetharamappa, J.: In vitro study on the interaction of mechanism of tricyclic compounds with bovine serum albumin. J. Pharm. Biomed. Anal. 35, 655-664 (2004)

25. Yan, H., Zhao, S.L., Yang, J.G., Zhu, X.D., Dai, G.L., Liang, H.D., Pan, F.Y., Weng, L.: Interaction between levamisole hydrochloride and bovine serum albumin and the influence of alcohol: spectra. J. Solution Chem. 38, 1183-1192 (2009)

26. Xu, M., Chen, F.J., Huang, L., Xi, P.X., Zeng, Z.Z.: Binding of rare earth metal complexes with an ofloxacin derivative to bovine serum albumin and its effect on the conformation of protein. J. Lumin. 131, 1557-1565 (2011)

27. Zhang, H.X., Huang, X., Mei, P., Gao, S.: Interaction between glyoxal-bis-(2-hydroxyanil) and bovine serum albumin in solution. J. Solution Chem. 37, 631-640 (2008)

28. Xu, H., Liu, Q.W., Wen, Y.Q.: Spectroscopic studies on the interaction between nicotinamide and bovine serum albumin. Spectrochim. Acta A 71, 984-988 (2008)

29. Bi, S.Y., Song, D.Q., Kan, Y.H., Xu, D., Tian, Y., Zhou, X., Zhang, H.Q.: Spectroscopic characterization of effective components anthraquinones in Chinese medicinal herbs binding with serum albumins Spectrochim. Acta A 62, 203-212 (2005)

30. Stryer, L.: Fluorescence energy transfer as a spectroscopic ruler. Ann. Rev. Biochem. 47, 819-846 (1978)

31. Gharagozlou, M., Boghaei, D.M.: Interaction of water-soluble amino acid Schiff base complexes with bovine serum albumin: fluorescence and circular dichroism studies. Spectrochim. Acta A 71, 1617-1622 (2008)

32. Zhang, H.X., Mei, P., Yang, X.X.: Optical, structural and thermodynamic properties of the interaction between tradimefon and serum albumin. Spectrochim. Acta A 72, 621-626 (2009)

33. Cheng, X.X., Lui, Y., Zhou, B., Xiao, X.H., Liu, Y.: Probing the binding sites and the effect of berbamine on the structure of bovine serum albumin. Spectrochim. Acta A 72, 922-928 (2009)

34. Lin, H., Lan, J.F., Guan, M., Sheng, F.L., Zhang, H.X.: Spectroscopic investigation of interaction between mangiferin and bovine serum albumin. Spectrochim. Acta A 73, 936-941 (2009) 